\title{
CLASSIFICATION OF NEW SPECTRAL LINES OF FE XVII OBSERVED IN SOLAR ACTIVE REGIONS
}

\author{
R. J. HUTCHEON \\ Dept. of Physics, University of Leicester, England
}

\begin{abstract}
Summary. This summary reports new emission lines of Fe XVII observed in two coronal active regions (McMath regions 12624, 12628). The lines were recorded by a Bragg plane crystal spectrometer collimated to $3^{\prime}$ FWHM, with a gypsum crystal scanning the wavelength range 8-14 $\AA$. This spectrometer, part of a three spectrometer package previously used by Parkinson $(1972,1973)$, was mounted aboard a Skylark rocket (SL 1206) launched from Woomera at 0535 UT on 26th November 1973. One of the active regions studied (12624) was observed about 60 minutes after the peak of an importance $-N$ class $\mathrm{CO}$ flare.
\end{abstract}

\section{TABLE I}

Measured and predicted wavelengths for newly identified Fe xvII transitions

Classification

Pair coupling notation

$$
\begin{array}{r}
2 s^{2} 2 p^{6}{ }^{1} S_{0}-2 s^{2} 2 p^{5}\left({ }^{2} P_{3 / 2}\right) 4 s[3 / 2]_{2} \\
2 p^{5}\left({ }^{2} P_{3 / 2}\right) 5 s[3 / 2]_{2} \\
2 p^{5}\left({ }^{2} P_{3 / 2}\right) 5 s[3 / 2]_{1} \\
2 p^{5}\left({ }^{2} P_{1 / 2}\right) 5 s[1 / 2]_{1} \\
2 p^{5}\left({ }^{2} P_{3 / 2}\right) 7 s[3 / 2]_{2} \\
2 p^{5}\left({ }^{2} P_{3 / 2}\right) 7 s[1 / 2]_{1} \\
2 p^{5}\left({ }^{2} P_{1 / 2}\right) 7 s[1 / 2]_{1} \\
2 p^{5}\left({ }^{2} P_{1 / 2}\right) 7 d[3 / 2]_{1} \\
2 p^{5}\left({ }^{2} P_{1 / 2}\right) 8 d[3 / 2]_{1}
\end{array}
$$

$$
\begin{aligned}
& \text { (upper level) } \\
& 2 p^{5} 4 s^{3} P_{2} \\
& 2 p^{5} 5 s^{3} P_{2} \\
& 2 p^{5} 5 s^{3} P_{1} \\
& 2 p^{5} 5 s^{1} P_{1} \\
& 2 p^{5} 7 s^{3} P_{2} \\
& 2 p^{5} 7 s^{3} P_{1} \\
& 2 p^{5} 7 s^{1} P_{1} \\
& 2 p^{5} 7 d^{1} P_{1} \\
& 2 p^{5} 8 d^{1} P_{1}
\end{aligned}
$$$$
\text { LS coupling notation }
$$

Wavelength

Predicted

Observed

12.696

11.422 )

11.415

11.282

10.545 )

10.543 \}

10.429

10.382

10.217

Observed
12.698
$11.419 \mathrm{~B}$
$11.285 \mathrm{~B}$
10.544
10.431
10.385
10.217

B: Blend (see text)

The new lines are listed in Table I with their measured and predicted wavelengths. The proposed classifications are given in the pair coupling notation as this is the most appropriate. (The statement by Kastner et al. (1967) that the LS coupling scheme best describes the $2 p^{5} 3 d$ terms in Fe Xvil does not apply to the other $2 p^{5}$ ns, nd terms). However since the LS designations of many Fe XVII terms appear in the literature, the corresponding LS classifications are also given to aid reference.

Each wavelength was measured twice, once in each active region, except the 10.431 $\AA$ and $10.544 \AA$ lines which were only observed in the decaying flare region. The measured wavelengths given for the other lines are the average of two values which generally differed by less than $0.005 \AA$. The final uncertainty is probably within $0.003 \AA$. 
The predicted wavelengths were extrapolated from previously known Fe XVII terms (Swartz et al., 1971; Tyren, 1938) by Ritz's Law (Edlen, 1964). To satisfy one requirement of this procedure the base and predicted terms were limited to those described by the same vector coupling scheme (pair coupling). The predicted wavelengths should be accurate to within $0.005 \AA$, the main source of uncertainty being that in the base wavelengths, and therefore agree satisfactorily with experiment. These identifications are supported further by the smooth decrease of intensities towards the series limit.

The $11.285 \AA$ and $11.419 \AA$ features coincide in wavelength with laboratory lines classified as Fe XVIII $2 p^{5}-2 p^{4} 4 d$ transitions by Swartz et al. (1971). The intensities of other Fe XVII and Fe XVIII lines observed in our results suggest that these features are blends with significant contributions from the Fe XVIII transitions mentioned above and the Fe XVII transitions shown in Table I. The lines between $10.2 \AA$ and $10.6 \AA$ may possibly be blended with currently unknown Fe XVIII $2 p^{5}-2 p^{4} 5 d$ transitions which are expected to lie in this region. It is intended to discuss this further in a future publication.

The lines at $10.217 \AA$ and $11.419 \AA$ in our scans may account for lines at $10.23 \AA$ and $11.41 \AA$ reported, but not identified, by Neupert et al. (1973). However, their $10.23 \AA$ feature may also be due to Ne x L $\beta$ (at $10.239 \AA$ ). These authors also report a line at $10.86 \AA$. This may be due to the Fe XVII transition $2 p^{6}{ }^{1} S_{0}-2 p^{5}\left({ }^{2} P_{3 / 2}\right)$ $6 s\left[\frac{1}{2}\right]_{1}$, for which we predict the wavelength $10.849 \AA$.

\section{References}

Edlen, B.: 1964, 'Atomic Spectra', in Handbuch der Physik 27, Ed. by S. Flügge, Springer-Verlag, Berlin, p. 80.

Kastner, S. O., Omidvar, K., and Underwood, J. H.: 1967, Astrophys. J. 148, 269.

Neupert, W. M., Swartz, M., and Kastner, S. O.: 1973, Solar Phys. 31, 171.

Parkinson, J. H.: 1972, Nature (Phys. Sci.) 236, 68.

Parkinson, J. H.: 1973, Astron. Astrophys. 24, 215.

Swartz, M., Kastner, S. O., Rothe, E., and Neupert, W. M.: 1971, J. Phys. B. 4, 1747.

Tyren, F.: 1938, Z. Phys. 111, 314. 